\title{
TWO VARIATIONAL APPROACHES FOR DOMAIN DECOMPOSITION PROBLEMS SOLVED BY SGBEM WITH NON-CONFORMING DISCRETIZATIONS
}

\author{
R. Vodička
}

Civil Engineering Faculty, Technical University of Košice, Slovakia

\author{
V. Mantič, F. París \\ School of Engineering, University of Seville, Seville, Spain
}

\begin{abstract}
Two original approaches for solution of elastic boundary value problems with domain decomposition (DDBVP) using the symmetric Galerkin boundary element method (SGBEM) are presented. Each approach is based on a variational principle, a difference between them consists in the treatment of the coupling conditions which connect the solutions through an interface. The developed computer codes are able to deal with curved interfaces in a domain decomposition problem discretized by non-matching meshes of linear elements along the interfaces. The effectiveness of the methods is documented by a numerically solved example.
\end{abstract}

Представлены два оригинальных подхода для решения краевых задач упругости с разделением областей с использованием симметричного метода граничных элементов Галеркина. Каждый подход основан на одном вариационном принципе, разница между ними состоит в использовании объединяющих условий, которые соединяют решения через интерфейс (множество-разделитель). Разработанные компьютерные коды способны работать с криволинейными интерфейсами в задачах декомпозиции области, дискретизированной несогласованной сеткой линейных элементов вдоль интерфейсов. Эффективность методов продемонстрирована на конкретном численно решенном примере.

PACS: 02.70.Dh; 02.60.Lj; 02.70.-c

\section{DOMAIN DECOMPOSITION}

Let us consider an elastic body defined by a domain $\Omega$ with a bounded Lipschitz boundary $\partial \Omega=\Gamma$. Let $\mathbf{n}$ denote the outward unit normal vector defined almost everywhere on the boundary $\Gamma$. We confine ourselves only to an analysis in $2 \mathrm{D}$ continuum (plain strain).

In the domain decomposition methods [2], we start with a split of the domain $\Omega$. For the sake of simplicity, let us consider a split into two non-overlapping parts $\Omega^{A}$ and $\Omega^{B}$, whose respective boundaries are denoted as $\Gamma^{A}$ and $\Gamma^{B}$, see Fig. 1. There also exists a common part of both boundaries, let us denote it by $\Gamma_{c}$. Considering the boundary conditions for displacements $\mathbf{u}^{\eta}$ and tractions $\mathbf{t}^{\eta}, \eta=A, B$ and the split of the boundary $\Gamma^{\eta}$ due to them, we can write the DDBVP for the Navier equation in the form

$$
\begin{aligned}
& c_{i j k l}^{\eta} u_{k, l j}^{\eta}(x)=c_{i j k l}^{\eta} \varepsilon_{k l, j}\left(\mathbf{u}^{\eta}(x)\right)=0, \quad x \in \Omega^{\eta}, \\
& u_{i}^{\eta}(x)=g_{i}^{\eta}(x), \quad x \in \Gamma_{u}^{\eta}, \\
& t_{i}^{\eta}(x)=\left(\mathcal{T}_{\mathbf{n}^{\eta}(x)}\right)_{i}\left(\mathbf{u}^{\eta}(x)\right)=h_{i}^{\eta}(x), \quad x \in \Gamma_{t}^{\eta}, \\
& t_{i}^{A}(x)=-t_{i}^{B}(x), \quad u_{i}^{A}(x)=u_{i}^{B}(x), \quad x \in \Gamma_{c},
\end{aligned}
$$




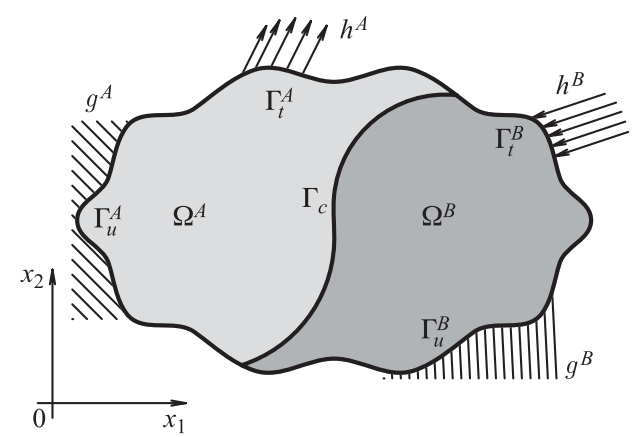

Fig. 1. A domain decomposition problem

with the elastic stiffness tensor $c_{i j k l}, i, j, k, l=1,2$, strain tensor $\varepsilon_{i j}$, traction operator $\mathcal{T}_{\mathbf{n}}$ and further with the natural coupling conditions providing compatibility of displacements and equilibrium of tractions (1d).

\section{VARIATIONAL FORMULATION}

The solution of (1) is constructed on a variational principle, based on the results of the one-domain SGBEM variational formulation introduced by Bonnet [1] and adapted for DDBVP by Vodicka et al. [4]. Let us introduce a functional of energy with Lagrange multipliers $E^{\lambda}\left(\mathbf{u}^{A}, \mathbf{u}^{B}, \boldsymbol{\lambda}_{u}, \boldsymbol{\lambda}_{t}\right)$, which is a function of displacements $\mathbf{u}^{A}, \mathbf{u}^{B}$ and of the Lagrange multipliers $\boldsymbol{\lambda}_{u}, \boldsymbol{\lambda}_{t}$. Physically, they correspond to some displacements and tractions at the interface. The functional $E^{\lambda}$ can be expressed in the following form:

$$
E^{\lambda}\left(\mathbf{u}^{A}, \mathbf{u}^{B}, \boldsymbol{\lambda}_{u}, \boldsymbol{\lambda}_{t}\right)=E_{t}^{A}\left(\mathbf{u}^{A}\right)+E_{t}^{B}\left(\mathbf{u}^{B}\right)+E_{c}^{\lambda}\left(\mathbf{u}^{A}, \mathbf{u}^{B}, \boldsymbol{\lambda}_{u}, \boldsymbol{\lambda}_{t}\right),
$$

where the functionals $E_{t}^{A}\left(\mathbf{u}^{A}\right)$ and $E_{t}^{B}\left(\mathbf{u}^{B}\right)$ introduce the total energy associated to the subdomains $\Omega^{A}$ and $\Omega^{B}$, respectively, with the exclusion of their interface boundary parts. Namely, taking $\eta=A, B$,

$$
E_{t}^{\eta}\left(\mathbf{u}^{\eta}\right)=\frac{1}{2} \int_{\Omega^{\eta}} \varepsilon_{i j}\left(\mathbf{u}^{\eta}\right) c_{i j k l}^{\eta} \varepsilon_{k l}\left(\mathbf{u}^{\eta}\right) d V-\int_{\Gamma_{t}^{\eta}} h_{i}^{\eta} u_{i}^{\eta} d S-\int_{\Gamma_{u}^{\eta}} t_{i}^{\eta}\left(u_{i}^{\eta}-g_{i}^{\eta}\right) d S .
$$

The last term in Eq. (2a) introduces a form of interface energy, modified by Lagrangemultipliers terms. It can be introduced by the relation

$$
\begin{aligned}
E_{c}^{\lambda}\left(\mathbf{u}^{A}, \mathbf{u}^{B}, \mathbf{t}_{c}^{A}, \mathbf{u}_{c}^{B}, \boldsymbol{\lambda}_{u}, \boldsymbol{\lambda}_{t}\right)=- & \int_{\Gamma_{c}} u_{i}^{B}\left(t_{i}^{A}+t_{i}^{B}\right) d S+ \\
& +\int_{\Gamma_{c}}\left(\lambda_{t}\right)_{i}\left(u_{i}^{B}-u_{i}^{A}\right) d S+\int_{\Gamma_{c}}\left(\lambda_{u}\right)_{i}\left(t_{i}^{A}+t_{i}^{B}\right) d S .
\end{aligned}
$$


The functions $\mathbf{t}^{A}$ and $\mathbf{t}^{B}$ represent the tractions of the displacement solutions $\mathbf{u}^{A}$ and $\mathbf{u}^{B}$, respectively, calculated via the traction operator $\mathcal{T}_{\mathbf{n}}$.

The interface energy introduces additional unknowns — Lagrange multipliers to explicitly set the interface conditions (1d). As will be shown in what follows, these unknowns can be eliminated. Namely, if we put $\boldsymbol{\lambda}_{u}$ equal to $\mathbf{u}^{B}$ and $\boldsymbol{\lambda}_{t}$ to $\mathbf{t}^{A}$, we obtain the following interface energy:

$$
E_{c}^{R}\left(\mathbf{u}^{A}, \mathbf{u}^{B}\right)=-\int_{\Gamma_{c}} t_{i}^{A}\left(u_{i}^{A}-u_{i}^{B}\right) d S
$$

which also reduces $E^{\lambda}$ in (2a) to a new energy functional $E^{R}\left(\mathbf{u}^{A}, \mathbf{u}^{B}\right)$.

Both introduced energy functionals can be used to solve the DDBVP (1), as their stationary point provides the problem solution. This fact can easily be observed considering vanishing variations of both functionals

$$
\begin{gathered}
\delta E^{\lambda}\left(\mathbf{u}^{A}, \mathbf{u}^{B}, \boldsymbol{\lambda}_{u}, \boldsymbol{\lambda}_{t} ; \delta \mathbf{u}^{A}, \delta \mathbf{u}^{B}, \delta \boldsymbol{\lambda}_{u}, \delta \boldsymbol{\lambda}_{t}\right)=0, \\
\delta E^{R}\left(\mathbf{u}^{A}, \mathbf{u}^{B} ; \delta \mathbf{u}^{A}, \delta \mathbf{u}^{B}\right)=0
\end{gathered}
$$

with virtual functions $\delta \mathbf{u}^{A}, \delta \mathbf{u}^{B}, \delta \boldsymbol{\lambda}_{u}, \delta \boldsymbol{\lambda}_{t}$.

We can eliminate the volume integrals and restrict the virtual displacements to those which satisfy the Navier equation (1a) applying an integral representation provided by the Somigliana displacement identity [3]:

$$
\delta u_{i}^{\eta}(x)=\int_{\Gamma^{\eta}} U_{i j}^{\eta}(x, y) \varphi_{j}^{\eta}(y) d_{y} S-\int_{\Gamma^{\eta}} T_{i j}^{\eta}(x, y) \psi_{j}^{\eta}(y) d_{y} S, \quad x \in \Omega^{\eta},
$$

where $U_{k l}^{\eta}$ is the fundamental solution of the Navier equation associated to the elastic material of $\Omega^{\eta}$ and $T_{k l}^{\eta}$ represents the fundamental tractions, obtained from the fundamental solution via the traction operator: $\mathbf{T}^{\eta}(x, y)=\left(\mathcal{T}_{\mathbf{n}^{\eta}(y)} \mathbf{U}^{\eta}(x, y)\right)^{T}, T$ denoting the transpose matrix.

A similar representation can be introduced for the tractions pertinent to the virtual displacements, namely

$$
\delta t_{i}^{\eta}(x)=\int_{\Gamma^{\eta}} T_{i j}^{\eta *}(x, y) \varphi_{j}^{\eta}(y) d_{y} S-\int_{\Gamma^{\eta}} D_{i j}^{\eta}(x, y) \psi_{j}^{\eta}(y) d_{y} S, \quad x \in \Omega^{\eta},
$$

with $\mathbf{T}^{\eta *}(x, y)=\mathcal{T}_{\mathbf{n}^{\eta}(x)} \mathbf{U}^{\eta}(x, y), \mathbf{D}^{\eta}(x, y)=\mathcal{T}_{\mathbf{n}^{\eta}(x)} \mathbf{T}^{\eta}(x, y)$ and $\mathbf{n}^{\eta}(x)$ being a normal to an auxiliary curve passing through the point $x$ at which the traction is evaluated.

The integral representations (4) and (5) can be, after a standard limit-to-the-boundary process (which originates jumps in some integrals), substituted into variations (3) and (after reordering the terms, change of the integration order, and introducing an operator notation for simplicity) a matrix form of the resulting boundary integral equation systems can be derived [4].

The boundary integral equation systems for searching the stationary point of the functionals $E^{\lambda}$ and $E^{R}$, can be written in a matrix-operator form

$$
\boldsymbol{\Phi}^{T} \mathcal{A X}=\boldsymbol{\Phi}^{T} \mathbf{B}
$$


which should be valid for any virtual vector function $\boldsymbol{\Phi}$. The shapes of the matrix operators depend on the chosen energy functional. The difference between the application of $E^{\lambda}$ and $E^{R}$ includes additional equations and additional unknowns for the formulation with Lagrange multipliers.

For the use with SGBEM, it is convenient to leave the system in the weak form as it is natural for Galerkin methods. Moreover, in the described form the property of the symmetry, which have both integral operators is clearly visible. This property will also be proper to the matrix obtained after discretization.

\section{AN EXAMPLE}

Let us consider a ring body (elastic constants: $G=10^{4} \mathrm{MPa}, \nu=0.25$ ) loaded by four point tensile forces $F$. Due to the symmetry, only a quarter of the whole ring will be considered for the numerical solution, see Fig. 2,a. For the numerical solution, both variational approaches, based on $E^{\lambda}$ and $E^{R}$, have been used, let us distinguish them by $\lambda$ and $R$, respectively. The results contain data of three different boundary element meshes along the interface: as the role of the subdomains $\Omega^{A}$ and $\Omega^{B}$ in the used variational formulation is different, the first mesh contains $N^{A}=48$ elements along $\Gamma_{c}$ on the $\Omega^{A}$-side and $N^{B}=112$ elements on the $\Omega^{B}$-side, the second mesh has the numbers of elements interchanged, i.e., $N^{A}=112, N^{B}=48$, and the third mesh has the same number of elements on both sides of the interface $\Gamma_{c}, N^{A}=N^{B}=48$. For the $R$ formulation only the first and the third meshes are considered, as the other case, when $N^{A}>N^{B}$ leads to numerically unstable results, see [4]. It should be noted that the non-conforming meshes along a curved $\Gamma_{c}$ leads to interpenetrations and gaps between the approximated subdomains, see Fig. 2, $b$. Nevertheless, even in such a situation the above numerical procedures are able to provide excellent approximations of the solution of the original problem.
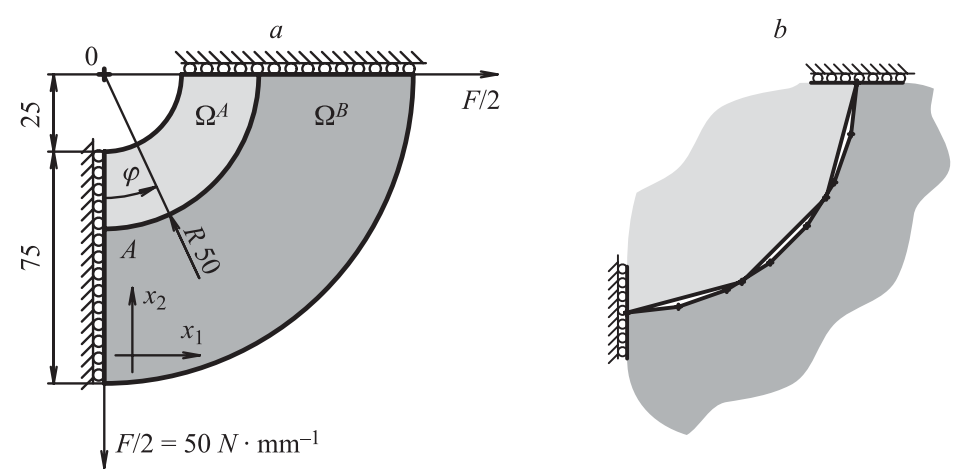

Fig. 2. The example: $a$ ) geometry description; $b$ ) a non-conforming mesh on the curved interface

The graphs contain the data obtained along the interface curve $A B$. Both displacements and tractions nicely fit the known analytical solution, nevertheless differences in the distribution of the errors appear, see Figs. 3, 4. While at the coarse meshes the distributions 
398 Vodička R., Mantič V., París F.
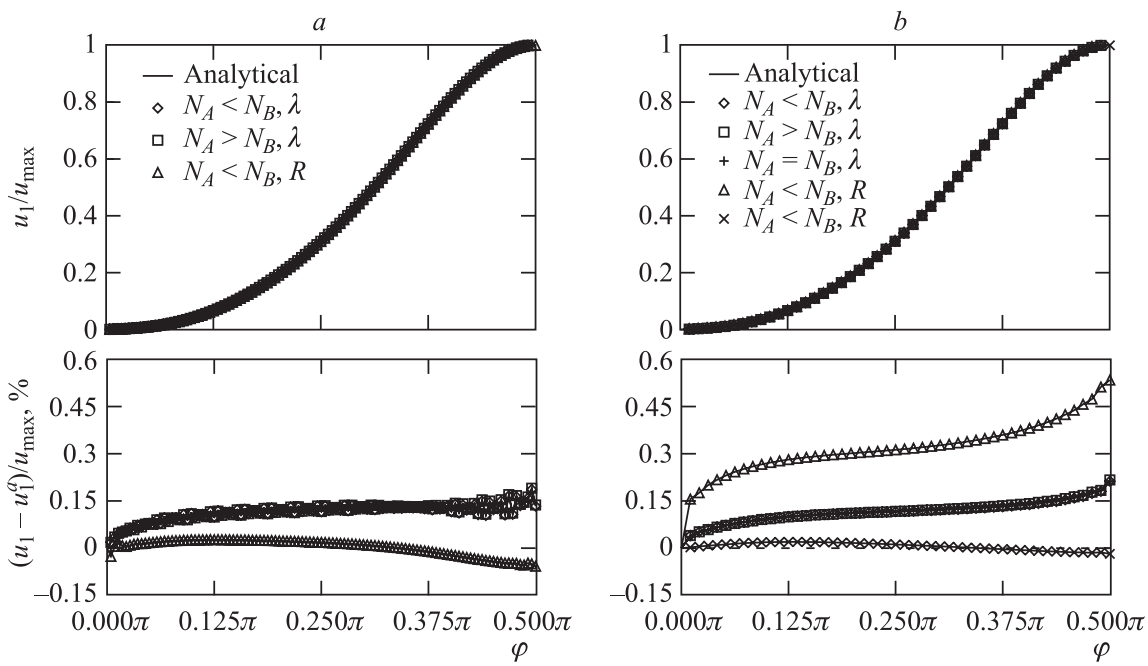

Fig. 3. Displacements and their errors: $a$ ) the fine mesh; $b$ ) the coarse mesh
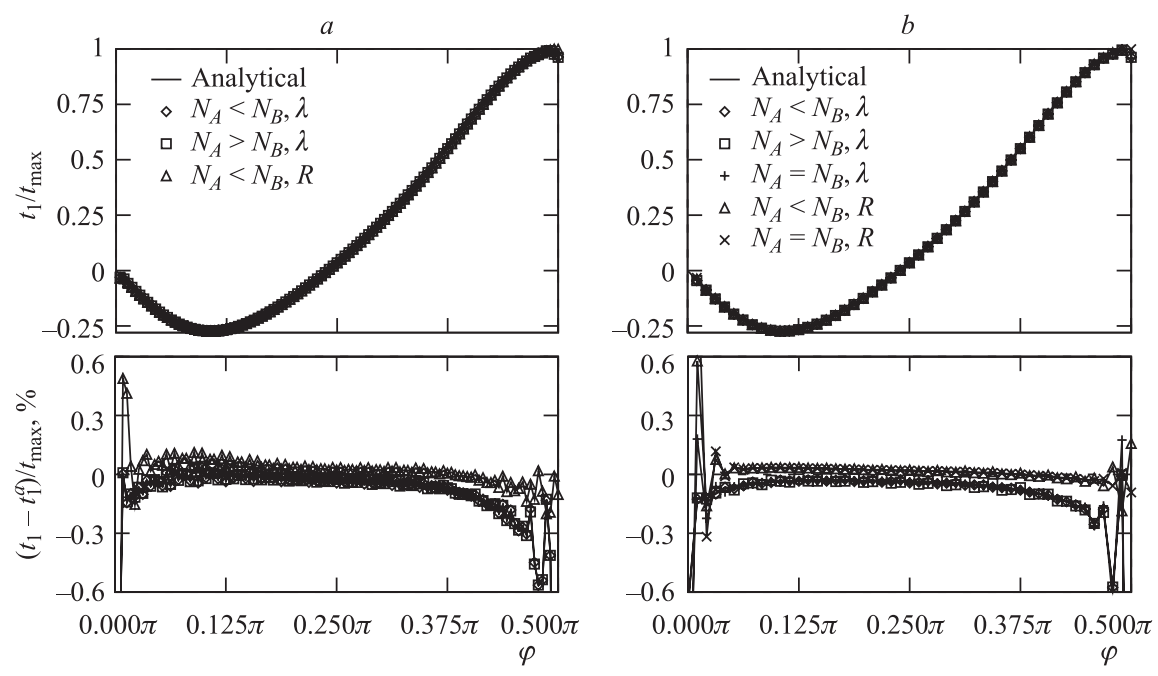

Fig. 4. Tractions and their errors: $a$ ) the fine mesh; $b$ ) the coarse mesh
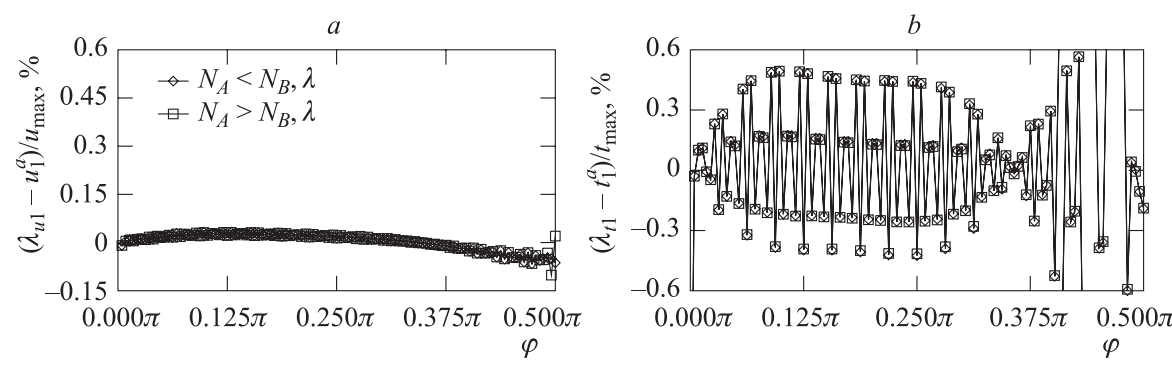

Fig. 5. Lagrange multipliers errors: a) $\lambda_{u}$; b) $\lambda_{t}$ 
are relatively smooth, the fine meshes show a zigzag character in the interior of the interface. The more apparent oscillations appear in the tractions close to the end points of the interface zone both for the coarse and fine meshes. The error distribution of the Lagrange multipliers of the $\lambda$ formulation in Fig. 5 even stresses the overall oscillatory behavior, especially $\lambda_{t}$ confirms this known property from some finite element formulations with Lagrange multipliers.

\section{CONCLUSIONS}

Two variational SGBEM approaches have been presented and their behaviours have been compared in an example. The first observation leads to a statement that both of them can be used with satisfactory results. Differences between them appear when we focus on the evaluation of the errors in the interface. Due to the character of the interface conditions and the type of their prescription, the numerical data at fine meshes always present some oscillations. The magnitude of the oscillation peaks depends on the type of the used mesh and on the form of the approach used.

The work is partially supported by VEGA, No.1/1006/04 and by Spanish Ministry of Science and Technology, No. MAT2003-03315.

\section{REFERENCES}

1. Bonnet M. // Engineering Analysis with Boundary Elements. 1995. V. 15. P.93-102.

2. Quarteroni A., Valli A. Domain Decomposition Methods for Partial Differential Equations. Oxford Univ. Press, 1999.

3. Ugodchicoff A. G., Khutoryanskiy N.M. Boundary Element Method in Solid Mechanics. Kazan Univ. Press, 1986 (in Russian).

4. Vodička R., Mantič V., París F. Numerical Methods in Continuum Mechanics // 4th Workshop on Trefftz Methods. CEACM, 2005. 